\title{
FÉLIX Y BENTO: DOS CASOS DE MASCULINIDAD A-RELACIONAL EN RESSURREIÇÃO Y DOM CASMURRO
}

\author{
Miquel Bota \\ California State University, Sacramento
}

Resumen: Este artículo presenta una nueva lectura de dos personajes masculinos de Joaquim Maria Machado de Assis: Félix, de su primera novela Ressurreição (I872), y Bento, de la novela Dom Casmurro (I899). Propone revisar estas novelas bajo la clave de la violencia de género para indagar en el maltrato psicológico que infligen a las mujeres con las que se vinculan emocionalmente, Capitu y Lívia, para explorar cómo representan la función de «hombre a-relacional» tal y como la describe Rainer Maria Rilke.

Palabras clave: literatura brasileña del siglo xix; Machado de Assis; estudios de género; Rainer Maria Rilke; masculinidad; hombre a-relacional.

\section{Félix and Bento: Two Cases of A-relational Masculinity in Ressurreição and Dom CASMurro}

Aвstract: This article presents a new reading of two works by Brazilian author Joaquim Maria Machado de Assis, his first novel Ressurreição (1872) and the later Dom Casmurro (I899). It reflects on two male protagonists, Félix and Bento, and the relationships they develop with their female partners, Capitu and Lívia. Through an exploration of the psychological abuse these two men inflict on their partners and how these men conform the concept of "arelational man" by Rainer Maria Rilke, it highlights the relevance of gender violence criticism in Machado's works.

Keywords: I9th-century Brazilian literature; Machado de Assis; gender studies; Rainer Maria Rilke; masculinity; arelational man.

En la nota previa a la edición de 1905, Machado de Assis (1998: 15) explicaba que Ressurreição, publicada por primera vez en 1872 , se reeditaba sin cambios ni mutaciones, salvo algunas correcciones gramaticales: «Este foi o meu primeiro romance, escrito aí vão muitos anos. Dado em nova edição, não lhe altero a composição nem o estilo, apenas troco dois ou três vocábulos, e faço tais ou quais correções de ortografia». Para entonces había publicado Dom Casmurro (I899) y era una figura consagrada en el panorama cultural brasileño. El paralelismo entre estas dos novelas, Ressurreição y Dom Casmurro, ha sido 
muy analizado por parte de la crítica (Gledson I984; Schwarz 200 I); sin embargo, su repercusión ha sido dispar: si bien Dom Casmurro se considera una obra cumbre del autor y de la literatura brasileña, Ressurreição, muy probablemente por formar parte del primer ciclo creativo de Machado de Assis, ha pasado por ser una buena primera novela, tal vez necesaria para crear la magistral Don Casmurro. Coincidiendo con la traducción al inglés de Karen Sotelino en 2013, críticos como José Luiz Passos (2007) y Roberto Schwarz (200I), entre otros, han reivindicado la entidad que Ressurreição tiene por sí misma. Es interesante que el propio autor, ya en la última etapa de su vida y en plena madurez intelectual y creativa, y treinta y tres años después de su primera publicación, decidiera no hacer cambio alguno en su primera novela. En el recién estrenado siglo xx, Ressurreição seguía manteniendo para su autor total vigencia en contenido.

El hecho de que se haya traducido por primera vez al inglés ciento cuarenta y un años después de su primera publicación invita a la reflexión: ¿Qué es lo que hace que ya en la segunda década del siglo Xxi se interpele, tanto desde dentro como desde fuera de Brasil, esta primera novela que hasta el momento había resonado con dignidad pero sin gloria en su devenir? Un nuevo significado complementario a las lecturas que hasta el momento han marcado el entendimiento del texto parece cobrar fuerza. Tal y como indica Antonio Cândido (I970: 13):

Nas obras dos grandes escritores é mais visível a polivalência do verbo literário. Elas são grandes porque extremamente ricas de significado, permitindo que cada grupo e cada época encontrem as suas obsessões e as suas necessidades de expressão. Por isso, as sucessivas gerações de leitores e críticos brasileiros foram encontrando níveis diferentes em, Machado de Assis, estimando-o por motivos diversos e vendo nele um grande escritor devido a qualidades por vezes contraditórias. O mais curioso é que provavelmente todas essas interpretações são justas, porque ao apanhar um ângulo não podem deixar de ao menos pressentir os outros.

Teniendo en cuenta la coyuntura social actual, en la que movimientos como el «women's march» o el «me too» canalizan las preocupaciones de una parte de la población, resulta sustancial realizar una lectura de género de la obra de Machado de Assis. Por ello, en mi lectura propongo que figuras masculinas como Félix y Bento ejemplifican, con su modo de razonar y actuar, la violencia de género, y representan la figura del maltratador, una entidad reco- 
nocida y denunciada en el tiempo presente pero social, cultural y mediáticamente ninguneada en el momento en el que Machado de Assis escribe sus novelas. El nuevo enfoque con que se leen estos textos del autor carioca como alegatos irónicos y muy sofisticados en contra del dominio masculino deriva en parte del modo en que estos personajes encarnan lo que Rainer Maria Rilke ha denominado «hombre a-relacional». Félix y Bento son claras muestras de la personificación de las propuestas de Rilke, que meditan sobre la incapacidad masculina para vincularse con lo femenino y la tendencia de los hombres a aislarse del mundo de lo afectivo/doméstico.

A pesar de no desplegar los juegos metaliterarios de Dom Casmurro, la historia de Félix y Lívia presenta retos e incertidumbres similares a los de Bento y Capitu. En las dos novelas, la voz narrativa presenta una historia de (des) amor desde un punto de vista absoluto. Los narradores pretenden, en ambos casos, una credibilidad categórica. El narrador de Ressurreição, a quien no se le identifica con el protagonista, aunque pretende ofrecer en sus reflexiones una descripción global de la historia, no consigue desembarazarse de sus juicios morales. De este modo, en la historia de Félix, la narración se centra en el cuestionamiento de la verdadera naturaleza de los sentimientos de Lívia y en su honestidad. En el caso de Dom Casmurro, el narrador es el propio Bento hablando bajo su alias «Dom Casmurro» (mote que, como él mismo explica, le ha puesto un joven poeta resentido a quien él no presta el menor interés). Aquí también se juzga la honradez de la mujer protagonista. La crítica no se cuestionó este hecho hasta mediados del siglo xx, cuando un sector de esta caracterizó a Bento como un celoso empedernido. Tal y como señala Silviano Santiago (2001: 66):

The reader, forgetting the thoughtful conscience of this man in his sixties would take the position of a judge and feel obliged to give a veredict on the narrator's phantasms, when in reality the only interest that Machado de Assis wants to awaken is in the moral person of Dom Casmurro. To sum up: the critics were interested in seeking the truth about Capitu, or the impossibility of having the truth about Capitu, when the only truth to be sought is Dom Casmurro.

En ambas historias, es la figura de la mujer la que se pone en tela de juicio. Una lectura irónica, sin embargo, hace que desconfiemos de esa aproximación. Otra vez lo que difiere en ambas historias es que en Ressurreição el narrador parece pasar cuentas sobre la verdadera naturaleza de Félix, a quien 
describe como un fracasado en las dos últimas frases del texto: «Não se contentando com a felicidade exterior que o rodeia, quer haver essa outra das afeições íntimas, duráveis e consoladoras. Não a há de alcançar nunca, porque o seu coração, se ressurgiu por alguns dias, esqueceu na sepultura o sentimento da confiança e a memoria das ilusões» (Machado de Assis 1998: 104). En Dom Casmurro, Bento, aunque autoproclame que vive en un estado de felicidad y complacencia, tampoco ha conseguido la resurrección de su corazón. Los dos protagonistas masculinos viven en soledad reivindicando lo acertado de su aislamiento afectivo y su rechazo a la vida familiar.

Desde su publicación hasta hace poco más de cincuenta años, lo que se pretendió evaluar y lo que puede ser reprensible socialmente es, en ambos casos, la conducta de la mujer. En ig6o la crítica Helen Caldwell da un giro en la interpretación de Dom Casmurro para hacer hincapié en que no hay que cuestionarse la inocencia de Capitu, sino la perversidad de Bento/Dom Casmurro, elemento que deja muy claro ya en el título de su trabajo: The Brazilian Othello of Machado de Assis. El propio Machado de Assis señala irónicamente esta relación cuando juega con el lector al titular el apartado I35 de la novela como «Otelo». La voluntad de Caldwell por fijar su atención en la figura del protagonista masculino, su entidad moral y en aquello que se omite en la narración ha permitido nuevas posibilidades de lectura, una nueva repercusión de las obras. Convertir las figuras de Bento y Félix como objeto de análisis, y concretamente en la idea de manipulación de su modo de obrar y de su discurso, permite examinar el texto desde esta otra perspectiva, muy ligada a la lectura contemporánea de la violencia de género en la que el maltrato psicológico se hace presente como elemento central. Desde este punto de vista, en ambas novelas se ejemplifica cómo dos varones adultos y de clase alta ejercen violencia emocional de género de un modo muy sutil pero efectivo. Siguiendo los postulados de Beverly Engel en The Emotionally Abused Woman: Overcoming Destructive Patterns and Reclaiming Yourself, puede entenderse el abuso como cualquier acto cuya finalidad es controlar a otra persona haciendo uso del miedo y la humillación. Con sus actitudes de duda, desconfianza y falta de apoyo, Bento y Félix ejercen su abuso de un modo «pasivo-agresivo», van minando el desarrollo psicológico de sus parejas, las cuales se ven atrapadas en una tela de araña muy bien trazada.

El momento y el lugar desde los que escribe Machado de Assis no ofrecen a la mujer mucho margen de actuación pública y la someten a la voluntad del hombre. Por tanto, deberán limitar sus acciones a la esfera privada a la que es- 
tán relegadas. Bajo esta premisa, una lectura literal focalizaría el análisis en su función en cuanto personas traidoras y ambiciosas, a la vez que idealistas y amorosas. Sin embargo, como ya se ha indicado, la crítica machadiana ha consensuado que es la ironía el tropo que orienta el rumbo de la interpretación de la obra de Machado de Assis. De este modo, tanto Bento como Félix van a trazar una trayectoria durante el transcurso de los eventos de sus historias, cuya finalidad va a ser expulsar a sus respectivas parejas del espacio doméstico compartido, relegándolas al ostracismo y al aislamiento social. Teniendo en cuenta, como se ha señalado, que no hay esfera pública para la mujer en el periodo en que se desarrollan las narraciones, impedirles un ambiente privado es ejercer sobre ellas violencia emocional, profesada de un modo tolerado y en parte fomentado bajo el sistema patriarcal en el que viven. Si se considera a Capitu y Lívia como mentirosas y ambiciosas, no se les puede recriminar a Bento y a Félix que las aparten de su lado y las condenen a la soledad.

Las historias de Bento en Dom Casmurro y Félix en Ressurreição tienen una afinidad evidente: ambas se tejen bajo un marco de duda y celos por parte de sus protagonistas respecto a las dos mujeres que, en teoría, se ganarán sus afectos sentimentales. Primer amor y esposa de Bento, Capitu representa un tipo de mujer no muy distante a lo que en apariencia hubiera sido Lívia si finalmente Félix se hubiera casado con ella. La importancia de las mujeres en la obra de Machado de Assis ya ha sido señalada por John Gledson (I984: 9): «Las mujeres con una extraña mezcla de idealismo y traición, amor y ambición, son fundamentales para algunas de las obras más exitosas y características del escritor», pero la verdadera naturaleza de su función no ha sido siempre entendida del mismo modo. Capitu ha despertado siempre mucho interés al representar una idea de mujer amiga/amante/traidora por excelencia que caracteriza a las grandes protagonistas decimonónicas. Lívia, sin embargo, ha suscitado menos interés. Como ya he señalado, en parte porque es la protagonista de la primera novela de Machado de Assis, de ese primer ciclo «menos brillante», pero también porque en apariencia está menos interesada en que la relación se legalice, no hay ningún rastro de responsabilidad en ella. El enfoque del narrador de Ressurreição hace que culpar a Lívia sea mucho más problemático.

En Dom Casmurro, Bento sospecha que su esposa le ha sido infiel con su mejor amigo, Ezequiel, y que este es en verdad el progenitor de su único hijo, llamado también Ezequiel. En Ressurreição, Félix margina a Lívia basándose en la duda, en si en verdad ella amó a su primer esposo, y en la maledicencia 
materializada en una carta escrita para desacreditarla. La duda sobre la honorabilidad de Lívia se sustenta en el modo en que la información le va siendo desvelada al lector, y se hace manifiesta la manipulación de un narrador que pretende ser objetivo. Un caso muy evidente de este condicionamiento lo recibe el lector en el episodio en el que Félix descubre que Lívia tiene un hijo de cinco años. La forma en que se produce el encuentro induce al lector a solidarizarse con el sentimiento de engaño que sufre Félix, como si Lívia le hubiera estado ocultando el hecho de que era madre, esperando el momento oportuno (cuando él ya estuviera completamente enamorado de ella) para hacerlo. Resulta relevante la manera en que, en el caso de Félix, el propio narrador refleja una mezcla de distancia e incomodidad emocional respecto a lo familiar, tal y como ocurre cuando conoce a Luís: «Durante esta cena, Félix parecerá completamente estranho a tudo que o rodeava. Não ouvia as repreensões da moça, nem a tagarelice da criança; ouvia-se a si mesmo. Contemplava aquele quadro com deleitosa inveja, e sentia pungir-lhe um remorso» (Machado de Assis 1998: 43).

En ambos casos, las acusaciones se basan en pruebas de escasa credibilidad, sospechas y tergiversaciones motivadas por un deseo no consciente de no implicarse emocionalmente. Llama la atención el esfuerzo y la pericia de Bento y Félix por justificar su conducta. Bento narra los elementos que considera centrales en su vida, desde la preadolescencia hasta el momento de la escritura de la narración cuando cuenta sesenta y pocos años. El tema central de la autobiografía de Dom Casmurro es la relación sentimental que este establece con su vecina Capitu, amiga de la infancia y esposa, llamada familiarmente Capitolina. El narrador se centra en las dificultades que ambos tienen que superar para que la relación llegue a consolidarse: desde que la madre de Bento rompa la promesa de consagrar a su hijo a la vida religiosa hasta superar la diferencia socioeconómica que hace de Capitu una candidata poco idónea para el joven. A pesar de todos esos avatares, la pareja conseguirá casarse con la bendición de doña Gloria. Si bien Bento, al recontar su historia, parece apuntar cierta opacidad en los actos de Capitu desde su más tierna infancia. De todos modos, una vez casados y al superar la dificultad inicial de engendrar un hijo, consiguen la felicidad. Junto al matrimonio formado por Ezequiel Escobar (el mejor amigo de Bento) y Sancha, y la hija de ambos, Capituzinha, vivirán en un escenario idílico que se enturbiará por las sospechas de Bento de la infidelidad de la esposa con Escobar. Bento no decide actuar hasta que considera que tiene pruebas fehacientes de la traición de su esposa. Parece que lo 
que no quiso considerar antes de la paternidad no puede desecharlo después de esta. Según el narrador, el niño presenta rasgos muy similares a los de Escobar. Con la muerte inesperada de Escobar, Bento confirma sus sospechas al observar a su esposa contemplando el cadáver, y decide enviar a madre e hijo a Suiza. Bento cuenta su historia cuando ambos, esposa e hijo, ya han muerto. De este modo, la novela, además de ser un buen reflejo de la sociedad carioca de finales del siglo XIX, cumpliendo la función de crítica social que Roberto Schwarz (200I) le atribuye a Machado de Assis, y también de ser un buen ejemplo de la habilidad metaliteraria con juegos constantes entre creador y lector, tal y como defiende Abel Barros Baptista (2003), va más allá, ahondando en cuestiones de violencia de género.

En el caso de Félix, la narración explica su casi matrimonio, algo que para él al final es un episodio más de su vida. La historia de Félix explica hechos que se iniciaron diez años atrás, cuando él tenía treinta y seis años. En este sentido, las dudas se materializarán en un periodo de tiempo mucho más breve. Desde el comienzo parece que todo juega a favor para que la relación entre los dos protagonistas de la historia vaya viento en popa. Félix es soltero y Lívia una viuda joven. Todo su entorno cree en la pertinencia de un matrimonio entre ambos.

Machado de Assis expone la incapacidad que tienen sus protagonistas masculinos, Bento y Félix, de ser felices bajo la idea de masculinidad que el patriarcado les ofrece, arruinando en su viaje la vida de aquellas mujeres que se enamoran de ellos. Tal y como explican R. W. Connel y James W. Messerschmidt (2005: I6): «To sustain a given pattern of hegemony requires the policing of man as well as the exclusion or discrediting of women». La dificultad de estos hombres para aceptar su emotividad (lo que consideran fragilidad), sus ambigüedades, los lleva a rechazar la fortaleza que, por el contrario, destaca en sus protagonistas femeninas. En realidad, lo que pone en evidencia es que, en el juego de roles de género, el varón se impone por su situación de dominio institucionalizada y por su incapacidad para abrirse a su emotividad.

En sus cartas a Lou Andreas-Salomé, Rainer Maria Rilke propone la figura del hombre a-relacional, al que define como una entidad transicional en la que están atrapados la mayoría de hombres, un hombre que vive en soledad emocional:

This man of the «new grain» has emerged, whose defining attribute is solitude. Since it is neither physically nor ontologically possible for any of us to be alone, 
this man is «going to pieces». When this «salutary» process of decomposition is complete, he will finally start learning how to love, and at a point in the future we will witness something that we not seen yet: the heterosexual couple. (Rilke 1969: 49)

Machado de Assis reflexiona principalmente desde la mente de sus protagonistas masculinos. Está interesado en describir todas las acciones que estos toman respecto a sus relaciones afectivas con las mujeres y las justificaciones morales sobre las que las apoyan. Tanto en el caso de Bento en Dom Casmurro como en el de Félix en Ressurreição, los hombres protagonistas echan a sus mujeres, sus familias, y evaden sus responsabilidades sociales, mostrando claramente que no están supeditados al qué dirán y conformando la idea de Rilke de que el hombre a-relacional de su tiempo escapa de la carga que para él representan la esposa y la familia: Bento al desterrar a Capitu y a su hijo Ezequiel, y Félix al no casarse con Lívia y, así, al no convertirse en padre adoptivo del hijo de esta.

En ese sentido, en el prólogo a la traducción de Ressurreição al inglés de Karen Sotelino, José Luiz Passos (2013: 9) expone que:

Contrary to the contemporary trend in Brazil, in his first novel Machado de Assis opted to depict the intimate conflict between two protagonists threatened by the shadow of past romantic experiences. Ressurrection develops a new theme: the scrutiny of the other from the point of view of someone who lives in self-deception —a bold idea for the pen of a novice in the genre.

Según Passos (2013: Io), Félix emula a Iago: «He (Félix) follows the suggestions of a rival for Lívia's affections, a man who leads him toward the realm of doubt, imitating Iago — the famous villain in Otelo».

Félix argumenta sus acciones de un modo malévolo: echándose la culpa de su desconfianza, no deja ninguna posibilidad a Lívia para que pueda dar la vuelta a lo que él quiere creer. Por lo tanto, al afirmar: «Eu não, minha querida Lívia, falta-me a primeira condição da paz interior: eu não creio na sinceridade dos outros» (Machado de Assis i998: 6I), Félix actúa más por condicionamiento interno que por presión social. En este sentido, la afirmación de Antonio Dimas, en el prólogo de la edición de Ressurreição de Ática, parece desestimar ese componente a-relacional propuesto por Rilke y que desde mi punto de vista es crucial para entender sus acciones: «Por subestimar Lívia e por superestimar-se é que Feliz se arrasa. Torturado pelo passado da noiva, Félix 
mata o futuro com a arma do ciúme do que já foi. Para ele, o principio social é mais importante do que o princípio do prazer e nisso ele se identifica mais uma vez com Werther em oposição a Dom Juan» (Dimas ig98: 8).

Lívia tiene, a ojos de la sociedad, un pasado prístino. Solo para Félix no es honesta. En realidad, la carta del rival solo sirve como excusa para justificar su deseo de no estar con ella. Nadie en el círculo social y afectivo de Félix tiene duda alguna sobre la honorabilidad de Lívia y su idoneidad para ser su esposa. El narrador de Ressurreição lo expone explícitamente en el último capítulo, «Hoje»:

O amor do médico teve dúvidas póstumas. A veracidade da carta que impediria o casamento, com o andar dos anos, não só lhe pareceu possível, mas até provável. Meneses disse-lhe um dia ter a prova cabal de que Luís Batista fora o autor da carta; Félix não recusou o testemunho nem lhe pediu a prova. O que ele interiormente pensava era que, suprimida a vilania de Luís Batista, não estava excluída a verossimilhança do fato, e bastava ela para lhe dar razão (Machado de Assis 1998: 104).

Es relevante el modo en que Machado de Assis, en su primera novela, presenta ya el tema de la desigualdad de género, mostrando cómo el hombre, atrapado en un rol machista, es incapaz de atender y entender la manera en que vive la relación amorosa la otra parte, y también es incapaz de escapar de la desconfianza y el escepticismo para entablar relaciones honestas y genuinas con otras personas. Cuando revisita el tema en Dom Casmurro, su punto de vista no ha cambiado; si acaso, su visión se ha vuelto más pesimista.

La incapacidad para ponerse en la piel de la otra persona se presenta en Ressurreição en una relación en la que la mujer, Lívia, es viuda, goza de experiencia, posición y respeto sociales. Lívia al final decide no casarse con Félix y criar sola a su hijo. El narrador, en el capítulo final, le dice al lector que Lívia en otro tiempo se hubiera retirado a un convento pero que en ese momento eso no se estila. Explica también que ha abandonado toda actividad pública guardando un buen recuerdo de Félix, a quien no ha olvidado, y que se dedica a criar a su hijo, que ya tiene quince años. El narrador puede manipular las palabras dibujando a una Lívia triste y resignada, sin embargo, el hecho es que se trata de una mujer independiente que no está sometida a los caprichos y volubilidades de nadie, y que está criando a su hijo: «Para consolo e companhia de sua velhice tem ela o filho, em cuja educação concentra todos os esforços. Luís possui as graças da mãe, apenas modificadas por uns toques varonis» (Machado de Assis I998: 104). 
Por otro lado, se decribe a Félix claramente como un hombre infeliz: «Félix é essencialmente infeliz. [...] Não a há d'alcançar nunca [la felicidad] porque o seu coração, se ressurgiu por alguns dias, esqueceu na sepultura o sentimento da confiança e a memoria das ilusões» (Machado de Assis i998: 104).

En el caso de Dom Casmurro, Capitu es más joven que Bento, y no goza de la misma posición social y económica. En cuanto se casa con él, toda su entidad social depende de ese vínculo. Una vez Bento decide echarla de su vida, no tiene modo alguno de reaccionar, no solo porque como esposa está supeditada legalmente a su marido, sino también porque no tiene independencia económica ni pertenece a una familia socialmente equiparable a la de su esposo, que pueda socorrerla. Mientras Lívia posee cierta agencia que le permite no caer bajo el yugo de Félix, Capitu no tiene escapatoria posible.

En Ressurreição, Machado de Assis presenta la posibilidad de parejas que conviven felizmente, como son los casos del coronel y dona Matilde, o también el de Meneses y Raquel. Resulta relevante la figura masculina de Meneses como contraria al modelo de Félix. Meneses procura escuchar, entender y respetar a las mujeres, como ya se muestra al inicio de la novela, en el segundo capítulo, titulado «Liquidação do ano velho», cuando en la conversación que mantiene con Félix le recrimina que haya terminado con su pareja del momento, Cecília:

—Fizeste mal em separar-te dela; Cecília amava-te.

-Meneses, disse Félix, eu nunca faço mal quando quebro uma cadeia: liberto-me. (Machado de Assis i998: 23)

Meneses termina felizmente casado con Raquel, que en principio estaba enamorada de Félix pero que consigue cambiar sus sentimientos. En este sentido, cabe señalar que Raquel es hija del coronel y dona Matilde, y goza de una posición y una seguridad que no tiene Capitu en Dom Casmurro. Bento no ofrece muchos detalles de la relación entre Escobar y Sancha; según su versión de los hechos no podía ser enteramente feliz si el amigo le ha sido infiel con Capitu. Sea o no eso cierto, Escobar muere y Sancha se queda viuda con una hija. Otra vez más, parece que las historias de Dom Casmurro ofrecen menos espacio al optimismo que las de Ressurreição, donde por lo menos nos presenta posibilidades de sosiego y felicidad si se toman las decisiones correctas. En los dos casos, desde la narración al final se hace evidente que hay una evocación de algo que no se consiguió, que no pudo ser. Tal y como expone José Luiz Passos (2013: 178): 
O narrador está em busca de uma ilusão. A evocação, o resgate mental dos 'tempos idos', caracteriza alguma das melhores narrativas de Machado. Em seus romances, a relação ansiosa com o tempo é principalmente uma relação do sujeito consigo; o seja, o narrador ou herói evoca o passado a fim de comprar-se com o que foi, e tornar-se com isso mais consciente do que poderia ter sido essa sensibilidade marcada pelo estilo do condicional hipotético, pela imaginação das possibilidades do sujeito comprimidas pelo fluxo inexorável de um tempo que a tudo diluí.

Para comprender la motivación de estos protagonistas, su deseo de vivir en soledad y alejarse de una relación heterosexual feliz y completa, y analizar su crueldad, resulta iluminador, como hemos visto, el concepto rilkiano de hombre a-relacional. La vigencia de los dos textos queda totalmente legitimada con la figura del maltratador y el retrato del sufrimiento de sus víctimas, dos mujeres que partiendo de lugares diferentes terminan siendo objeto de manipulación y violencia.

\section{BiBLIOGRAFÍA}

Baptista, Abel Barros (2003). A formação do nome. Campinas: Unicamp.

Caldwell, Helen (1960). The Brazilian Othello of Machado de Assis. Los Angeles: University of California Press.

CÂndido, Antonio (1970). Vários Escritos. São Paulo: Duas Cidades.

Connel, R.W.; Messerschmidt, James W (2005). «Hegemonic Masculinity: Rethinking the Concept». Gender and Society, vol. 19, n. ${ }^{\circ}$ 6, 829-859.

Dimas, Antonio (ed.) (1998). Ressurreição. São Paulo: Editora Ática.

Engel, Beverly (1992). The Emotionally Abused Woman; Overcoming Destructive Patterns and Reclaiming Yourself. New York: Fawcet.

Gledson, John (1984). «Machado de Assis between Romance and Satire A Parasita Azul». Salvador Bacarisse et al. (dir.). What's Past Is Prologue: A Collection of Essays in Honour of L. J. Woodward. Edinburgh: Scottish Academic Press, 57-64.

Machado de Assis, Joaquim Maria ([ı99] 2016). Dom Casmurro. São Paulo: Editora Carambaia.

Machado de Assis, Joaquim Maria ([1905] 1998). Ressurreição. Antonio Dimas (ed.). São Paulo: Editora Ática.

Machado de Assis, Joaquim Maria (2013). Resurrection. Trad. Karen Sotelino. Pittsburgh: Latin American Literary Review Press.

Passos, José Luiz (2007). Machado de Assis: o romance com pessoas. São Paulo: Editora da Universidade de São Paulo. 
Passos, José Luiz (ed.) (2013). Resurrection. Trad. Karen Sotelino. Pittsburgh: Latin American Literary Review Press.

Rilke, Rainer Maria ([1945] I969). Letters, I892-I9ıo. Trad. Jane Bannard Greene y M. D. Herter Norton. Nueva York: Norton.

Santiago, Silviano (200I). «The Rethoric of Verisimilitude». Ana Lúcia Gazzola (ed.). The Space in-Between. Essays on Latin American Culture. Durham: Duke University Press, $64-78$.

Schwarz, Roberto ([1990] 200I). Um mestre na periferia do capitalismo: Machado de Assis. São Paulo: Duas Cidades. 